\title{
A novel dual reciprocity boundary element formulation for two-dimensional transient convection-diffusion-reaction problems with variable velocity
}

\author{
Salam A. AL-Bayatia , Luiz C. Wrobel ${ }^{\mathrm{a}, \mathrm{b}, *}$ \\ ${ }^{a}$ College of Engineering, Design and Physical Sciences, Brunel University London, Uxbridge, UB8 3PH, UK \\ ${ }^{b}$ Institute of Materials and Manufacturing, Brunel University London, Uxbridge, UB8 3PH, UK
}

\begin{abstract}
This paper describes a new formulation of the dual reciprocity boundary element method (DRBEM) for two-dimensional transient convection-diffusion-reaction problems with variable velocity. The formulation decomposes the velocity field into an average and a perturbation part, with the latter being treated using a dual reciprocity approximation to convert the domain integrals arising in the boundary element formulation into equivalent boundary integrals. The integral representation formula for the convection-diffusion-reaction problem with variable velocity is obtained from the Green's second identity, using the fundamental solution of the corresponding steady-state equation with constant coefficients. A finite difference method (FDM) is used to simulate the time evolution procedure for solving the resulting system of equations. Numerical applications are included for three different benchmark examples for which analytical solutions are available, to establish the validity of the proposed approach and to demonstrate its efficiency. Finally, results obtained show that the DRBEM results are in excellent agreement with the analytical solutions and do not present oscillations or damping of the wave front, as it appears in other numerical techniques.
\end{abstract}

Keywords: DRBEM; Transient convection-diffusion-reaction; Variable velocity; Radial basis functions;

Time-marching schemes

\section{Introduction}

The solution of convection-diffusion-reaction problems is a difficult task for all numerical methods because of the nature of the governing equation, which includes first-order and second-order partial derivatives in space 1, 2, 3, 4, 5. The convection-diffusion equation is the basis of many physical and chemical phenomena,

5 and its use has also spread in economics, financial forecasting and other fields [6]. The dual reciprocity boundary element method (DRBEM), initially applied to transient heat conduction problems by Wrobel et al. [7, interprets the time derivative in the diffusion equation as a body force and employs the fundamental solution to the corrsponding steady-state equation to generate a boundary integral equation. When the steady-state fundamental solution is used in the DRBEM to approximate transient convection-diffusion problems, other techniques should be employed to approximate the solution's functional dependence on the

\footnotetext{
${ }^{*}$ Corresponding author,

Email addresses: Salam.AL-Bayati@brunel.ac.uk (Salam A. AL-Bayati), Luiz.Wrobel@brunel.ac.uk (Luiz C. Wrobel )
} 
temporal variables. Aral and Tang [8] used the fundamental solution of the Laplace equation, but made use of a secondary reduction process, called SR-BEM, to arrive at a boundary-only formulation. They presented the results of transient convection-diffusion problems with or without first order chemical reaction for low to moderate Péclet numbers. Martin [9] proposed a Schwartz waveform relaxation algorithm for the unsteady diffusive-convective equation, which uses domain decomposition methods and applies the iterative algorithm directly to the time-dependent problem. Partridge and Sensale [10] have used the method of fundamental solution with dual reciprocity and subdomain approach to solve convection-diffusion problems. The time integration scheme is the finite difference method (FDM) with a relaxation procedure, which is iterative in nature and needs a carefully selected time increment. Regarding the DRBEM formulation presented in this work, a backward finite difference scheme is adopted, Smith [11].

In this article, the DRBEM is also employed to discretise the spatial partial derivatives in the twodimensional diffusive-convective-reactive type problem. Thus, the problem is ultimately described in terms of boundary values only, consequently reducing its dimensionality by one [12. We use the fundamental solution to the steady-state convection-diffusion-reaction equation and transform the domain integral arising from the time derivative term using a set of coordinate functions and particular solutions which satisfy the associated non-homogeneous steady-state convection-diffusion-reaction problem. Further, only a simple set of cubic radial basis functions has been previously used in this formulation. We consider two other sets of coordinate functions, non-augmented thin plate spline (TPS) and multiquadric (MQ) radial basis functions, and analyse their performance in conjunction with order of time integration algorithms for convectiondiffusion-reaction problems. This work also focuses on the search for the optimal shape parameter when utilising the multiquadric radial basis function (MQ-RBF). This is due to the lack of information on choosing the best shape parameter, forcing the user having to make an 'ad-hoc' decision. Recent numerical experiments available in the literature, nevertheless, showed that the MQ-RBF has shown great potential when dealing with complicated PDEs in two dimensions if an adequate shape value is provided.

A brief outline of the rest of this paper is as follows. Section 2 reviews the representation of convectiondiffusion- reaction. Section 3 derives the boundary element formulation of the governing equation using the steady-state fundamental solution of the corresponding equation. In sections 4 and 5 , the DRBEM formulation and its discretisation are developed for the 2D transient convection-diffusion-reaction problem. A two-level time marching procedure for the proposed model is implemented in section 6 . Section 7 gives the description of the coordinate functions and the choice of the three radial basis functions. Section 8 compares and investigates the solution profiles for the present numerical experiments with the analytical solution of the tested cases. Computational aspects are included to demonstrate the performance of the approach in section 9. Finally, some conclusions and remarks are provided in the last section. 


\section{Convection-diffusion-reaction equation}

The two dimensional transient convection-diffusion-reaction problem over a domain $\Omega$ in $\mathbb{R}^{2}$ bounded by a boundary $\Gamma$, for isotropic materials, is governed by the following PDE:

$$
D \nabla^{2} \phi(x, y)-v_{x}(x, y) \frac{\partial \phi(x, y)}{\partial x}-v_{y}(x, y) \frac{\partial \phi(x, y)}{\partial y}-k \phi(x, y)=\frac{\partial \phi(x, y)}{\partial t},(x, y) \in \Omega, t>0
$$

In Eq.(1), $\phi$ represents the concentration of a substance, treated as a function of space and time. The velocity components $v_{x}$ and $v_{y}$ along the $x$ and $y$ directions and assumed to vary in space. Besides, $D$ is the diffusivity coefficient and $k$ represents the first-order reaction constant or adsorption coefficient. The boundary conditions are

$$
\begin{gathered}
\phi=\bar{\phi} \quad \text { over } \Gamma_{D} \\
q=\frac{\partial \phi}{\partial n}=\bar{q} \quad \text { over } \Gamma_{N},
\end{gathered}
$$

where $\Gamma_{D}$ and $\Gamma_{N}$ are the Dirichlet and Neumann parts of the boundary with $\Gamma=\Gamma_{D} \cup \Gamma_{N}$, and $\Gamma_{D} \cap \Gamma_{N}=0$ (see Fig. 1). The initial condition over the domain $\Omega$ is

$$
\phi(x, y, t=0)=\phi_{0}(x, y), \quad(x, y) \in \Omega
$$

The parameter that describes the relative influence of the convective and diffusive components is called the Péclet number, Pé $=|v| L / D$, where $v=\left(v_{x}^{2}+v_{y}^{2}\right)^{1 / 2}$ is the velocity field and $L$ is a characteristic length of the domain. For small values of Pé, Eq. (1) behaves as a parabolic differential equation in time, while for large values the equation becomes more like hyperbolic. These changes in the structure of the PDE according to the values of the Péclet number have significant effects on its numerical solution.

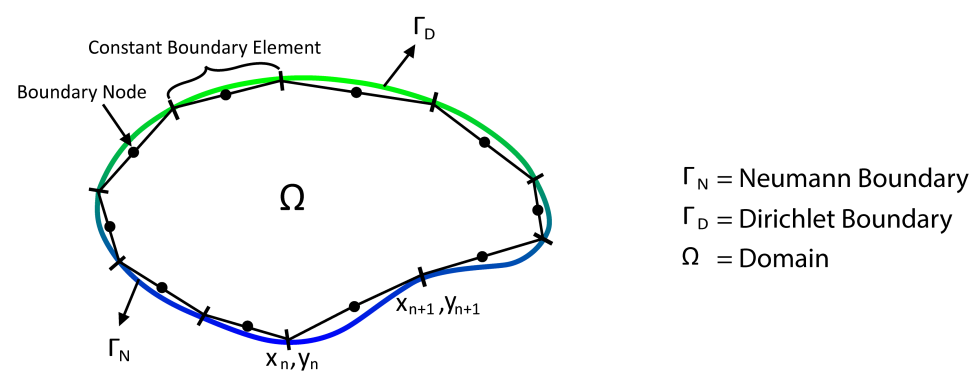

Figure 1: Definition of domain, boundary, and constant elements

50

\section{Boundary element formulation of transient convection-diffusion-reaction problems using steady-state fundamental solution}

Let us consider a region $\Omega \subset \mathbb{R}^{2}$ bounded by a piecewise smooth boundary $\Gamma$. The transport of $\phi$ in the presence of a reaction term is governed by the two-dimensional transient convection-diffusion-reaction Eq. (1). 
The variable $\phi$ can be interpreted as temperature for heat transfer problems, concentration for dispersion problems, etc, and will be herein referred to as a potential. For the sake of obtaining an integral equation equivalent to the above PDE, a fundamental solution of Eq.(1) is necessary. However, fundamental solutions are only available for the case of constant velocity fields. At this stage, the variable velocity components $v_{x}=v_{x}(x, y)$ and $v_{y}=v_{y}(x, y)$ are decomposed into average (constant) terms $\bar{v}_{x}$ and $\bar{v}_{y}$, and perturbations $P_{x}=P_{x}(x, y)$ and $P_{y}=P_{y}(x, y)$, such that

$$
v_{x}(x, y)=\bar{v}_{x}+P_{x}(x, y), v_{y}(x, y)=\bar{v}_{y}+P_{y}(x, y)
$$

Now, we can re-write Eq.(1) to take the form

$$
D \nabla^{2} \phi(x, y)-\bar{v}_{x}(x, y) \frac{\partial \phi(x, y)}{\partial x}-\bar{v}_{y}(x, y) \frac{\partial \phi(x, y)}{\partial y}-k \phi(x, y)=\frac{\partial \phi(x, y)}{\partial t}+P_{x} \frac{\partial \phi(x, y)}{\partial x}+P_{y} \frac{\partial \phi(x, y)}{\partial y} .
$$

Next, one can transform the differential equation (6) into an equivalent integral equation as follows [12]

$$
\begin{aligned}
\phi(\xi) & -D \int_{\Gamma} \phi^{*} \frac{\partial \phi}{\partial n} d \Gamma+D \int_{\Gamma} \phi \frac{\partial \phi^{*}}{\partial n} d \Gamma+\int_{\Gamma} \phi \phi^{*} \bar{v}_{n} d \Gamma \\
& =-\int_{\Omega}\left[\frac{\partial \phi}{\partial t}+\left(P_{x} \frac{\partial \phi}{\partial x}+P_{y} \frac{\partial \phi}{\partial y}\right)\right] \phi^{*} d \Omega, \xi \in \Omega
\end{aligned}
$$

where $\bar{v}_{n}=v . n, n$ is the unit outward normal vector and the dot stands for scalar product and $v=\left(v_{x}, v_{y}\right)$. In the above equation, $\phi^{*}$ is the fundamental solution of the steady-state convection-diffusion-reaction equation with constant coefficients. For two-dimensional problems, $\phi^{*}$ is given by

$$
\phi^{*}(\xi, \chi)=\frac{1}{2 \pi D} e^{-\left(\frac{\bar{v} \cdot r}{2 D}\right)} K_{0}(\mu r)
$$

where

$$
\mu=\left[\left(\frac{|\bar{v}|}{2 D}\right)^{2}+\frac{k}{D}\right]^{\frac{1}{2}}, \bar{v}=\left(\bar{v}_{x}, \bar{v}_{y}\right)
$$

in which $\xi$ and $\chi$ are the source and field points, respectively, and $r$ is the modulus of $\mathbf{r}=|\chi-\xi|$, the distance vector between the source and field points. The derivative of the fundamental solution with respect to the outward normal is given by

$$
\frac{\partial \phi^{*}}{\partial n}=\frac{1}{2 \pi D} e^{-\left(\frac{\bar{v} \cdot r}{2 D}\right)}\left[-\mu K_{1}(\mu r) \frac{\partial r}{\partial n}-\frac{\bar{v}_{n}}{2 D} K_{0}(\mu r)\right]
$$

In the above, $K_{0}$ and $K_{1}$ are Bessel functions of second kind, of orders zero and one, respectively. The exponential term is responsible for the inclusion of the correct amount of 'upwind' into the formulation [13. Eq. (7) is valid for source points $\xi$ inside the domain $\Omega$. A similar expression can be obtained, by implementing Green's second identity and a limit analysis, for source points $\xi$ on the boundary $\Gamma$, in the form

$$
c(\xi) \phi(\xi)-D \int_{\Gamma} \phi^{*} \frac{\partial \phi}{\partial n} d \Gamma+D \int_{\Gamma} \phi \frac{\partial \phi^{*}}{\partial n} d \Gamma+\int_{\Gamma} \phi \phi^{*} \bar{v}_{n} d \Gamma
$$




$$
=-\int_{\Omega} \frac{\partial \phi}{\partial t} \phi^{*} d \Omega-\int_{\Omega}\left(P_{x} \frac{\partial \phi}{\partial x}+P_{y} \frac{\partial \phi}{\partial y}\right) \phi^{*} d \Omega, \xi \in \Gamma
$$

in which $c(\xi)$ is a function of the internal angle the boundary $\Gamma$ makes at point $\xi$.

\section{DRBEM formulation for transient convection-diffusion-reaction problems}

In this section, we will discuss the transformation of the domain integral in Eqs.(7) and (11), and the DRBEM will be implemented twice to approximate the two domain integrals appearing in this formulation, first the domain integral of the time derivative, and second the domain integral related to the velocity perturbation parts. Now, we start by expanding the time-derivative $\frac{\partial \phi}{\partial t}$ in the form

$$
\frac{\partial \phi(x, y, t)}{\partial t}=\sum_{i=1}^{n} f_{i}(x, y) \alpha_{1, i}(t)
$$

The above series involves a set of known coordinate functions $f_{i}$ and a set of unknown time-dependent coefficients $\alpha_{1, i}$. With this approximation, the first domain integral in Eq. (11) becomes

$$
\int_{\Omega} \frac{\partial \phi}{\partial t} \phi^{*} d \Omega=\sum_{i=1}^{n} \alpha_{1, i} \int_{\Omega} f_{i} \phi^{*} d \Omega
$$

The next step is to consider that, for each function $f_{i}$, there exists a related function $\psi_{i}$ which is a particular solution of the equation:

$$
D \nabla^{2} \psi-v_{x} \frac{\partial \psi}{\partial y}-v_{y} \frac{\partial \psi}{\partial x}-k \psi=f
$$

Now, the domain integral (13) can be recast in the form:

$$
\int_{\Omega} \frac{\partial \phi}{\partial t} \phi^{*} d \Omega=\sum_{k=1}^{M} \alpha_{1, k} \int_{\Omega}\left(D \nabla^{2} \psi_{k}-\bar{v}_{x} \frac{\partial \phi_{k}}{\partial y}-\bar{v}_{y} \frac{\partial \phi_{k}}{\partial x}-k \psi_{k}\right) \phi^{*} d \Omega
$$

Substituting expression (15) into [11, and applying integration by parts to the right side of the resulting equation and doing some simplifications, one finally arrives at a boundary integral equation of the form

$$
\begin{gathered}
c(\xi) \phi(\xi)-D \int_{\Gamma} \phi^{*} \frac{\partial \phi}{\partial n} d \Gamma+D \int_{\Gamma} \phi \frac{\partial \phi^{*}}{\partial n} d \Gamma+\int_{\Gamma} \phi \phi^{*} \bar{v}_{n} d \Gamma \\
=\sum_{k=1}^{M} \alpha_{1, k}\left[c(\xi) \psi_{k}(\xi)-D \int_{\Gamma} \phi^{*} \frac{\partial \psi_{k}}{\partial n} d \Gamma+D \int_{\Gamma}\left[\left(\frac{\partial \phi^{*}}{\partial n}+\frac{\bar{v}_{n}}{D} \phi^{*}\right) \psi_{k}\right] d \Gamma\right] \\
-\int_{\Omega}\left(P_{x} \frac{\partial \phi_{k}}{\partial x}+P_{y} \frac{\partial \phi_{k}}{\partial y}\right) \phi^{*} d \Omega, \xi \in \Gamma
\end{gathered}
$$




\section{Space-time discretisation of the $2 \mathrm{D}$ convection-diffusion-reaction model}

To discretise the spatial domain, boundary elements were employed. The integrals over the boundary are approximated by a summation of integrals over individual boundary elements. For the numerical solution of the problem, Eq. (16) is discretised in the form

$$
\begin{gathered}
c_{i} \phi_{i}-D \sum_{j=1}^{N} \int_{\Gamma_{j}} \phi^{*} \frac{\partial \phi}{\partial n} d \Gamma+D \sum_{j=1}^{N} \int_{\Gamma_{j}}\left(\frac{\partial \phi^{*}}{\partial n}+\frac{\bar{v}_{n}}{D} \phi^{*}\right) \phi d \Gamma= \\
=\sum_{k=1}^{M} \alpha_{1, k}\left[c_{i} \psi_{i k}(\xi)-D \sum_{j=1}^{N} \int_{\Gamma_{j}} \phi^{*} \frac{\partial \psi_{k}}{\partial n} d \Gamma+D \sum_{j=1}^{N} \int_{\Gamma_{j}}\left[\left(\frac{\partial \phi^{*}}{\partial n}+\frac{\bar{v}_{n}}{D} \phi^{*}\right) \psi_{k} d \Gamma\right]\right] \\
-\int_{\Omega}\left(P_{x} \frac{\partial \phi_{k}}{\partial x}+P_{y} \frac{\partial \phi_{k}}{\partial y}\right) \phi^{*} d \Omega .
\end{gathered}
$$

where the index $i$ means the values at the source point $\xi$ and $N$ elements have been employed. The domain integral on the right-hand-side prevents us from obtaining a boundary-only equation.

Now, in order to obtain a boundary integral which is equivalent to the domain integral in expressions (16) and (17), a dual reciprocity approximation is implemented 2. Applying this to the domain integral of Eq. (17), the expression will be expanded in the form

$$
P_{x}(x, y) \frac{\partial \phi}{\partial x}+P_{y}(x, y) \frac{\partial \phi}{\partial y}=\sum_{k=1}^{N} \alpha_{2, k}(t) f_{k} .
$$

Expression 180 contains two diagonal matrices $P_{x}=\left(P_{x}\left(x_{i}, y_{i}\right) \delta_{i, j}\right)_{i, j=\overline{1, M}}$ and $P_{y}=\left(P_{y}\left(x_{i}, y_{i}\right) \delta_{i, j}\right)_{i, j=1, M}$ while $\frac{\partial \phi}{\partial x}=\left(\frac{\partial \phi\left(x_{i}, y_{i}\right)}{\partial x}\right)_{i=\overline{1, M}}^{T}$ and $\frac{\partial \phi}{\partial y}=\left(\frac{\partial \phi\left(x_{i}, y_{i}\right)}{\partial x}\right)_{i=\overline{1, M}}^{T}$ are column vectors and $\delta_{i, j}$ is the Kronecker delta symbol. Integrating Eq. 18 we obtain

$$
\int_{\Omega}\left(P_{x} \frac{\partial \phi}{\partial x}+P_{y} \frac{\partial \phi}{\partial y}\right) \phi^{*} d \Omega=\sum_{k=1}^{M} \alpha_{2, k}(t) \int_{\Omega} f_{k} \phi^{*} d \Omega .
$$

Now, substituting Eq.197 into (17), we obtain

$$
\begin{gathered}
c_{i} \phi_{i}-D \sum_{j=1}^{N} \int_{\Gamma_{j}} \phi^{*} \frac{\partial \phi}{\partial n} d \Gamma+D \sum_{j=1}^{N} \int_{\Gamma_{j}}\left(\frac{\partial \phi^{*}}{\partial n}+\frac{\bar{v}_{n}}{D} \phi^{*}\right) \phi d \Gamma \\
=\sum_{k=1}^{M} \alpha_{1, k}\left[c_{i} \psi_{i k}(\xi)-D \sum_{j=1}^{N} \int_{\Gamma_{j}} \phi^{*} \frac{\partial \psi_{k}}{\partial n} d \Gamma+D \sum_{j=1}^{N} \int_{\Gamma_{j}}\left[\left(\frac{\partial \phi^{*}}{\partial n}+\frac{\bar{v}_{n}}{D} \phi^{*}\right) \psi_{k} d \Gamma\right]\right] \\
-\sum_{j=1}^{M} \alpha_{2, j}(t) \int_{\Omega} f_{k} \phi^{*} d \Omega
\end{gathered}
$$


The next step is to consider that, for each function $f_{k}$, there exists a related function $\psi_{k}$ which represents the particular solution as in Eq.(14). We get

$$
\int_{\Omega}\left(P_{x} \frac{\partial \phi}{\partial x}+P_{y} \frac{\partial \phi}{\partial y}\right) \phi^{*} d \Omega=\sum_{k=1}^{M} \alpha_{2, k} \int_{\Omega}\left(D \nabla^{2} \psi_{k}-\bar{v}_{x} \frac{\partial \phi_{k}}{\partial y}-\bar{v}_{y} \frac{\partial \phi_{k}}{\partial x}-k \psi_{k}\right) \phi^{*} d \Omega .
$$

75 Substituting Eq.21) into expression (16), and applying integration by parts to the domain integral of the resulting equation, one finally arrives at a boundary integral equation of the form

$$
\begin{gathered}
c_{i} \phi_{i}-D \sum_{j=1}^{N} \int_{\Gamma_{j}} \phi^{*} \frac{\partial \phi}{\partial n} d \Gamma+D \sum_{j=1}^{N} \int_{\Gamma_{j}}\left(\frac{\partial \phi^{*}}{\partial n}+\frac{\bar{v}_{n}}{D} \phi^{*}\right) \phi d \Gamma \\
=\sum_{k=1}^{M} \alpha_{1, k}\left[c_{i} \psi_{i k}(\xi)-D \sum_{j=1}^{N} \int_{\Gamma_{j}} \phi^{*} \frac{\partial \psi_{k}}{\partial n} d \Gamma+D \sum_{j=1}^{N} \int_{\Gamma_{j}}\left[\left(\frac{\partial \phi^{*}}{\partial n}+\frac{\bar{v}_{n}}{D} \phi^{*}\right) \psi_{k} d \Gamma\right]\right] \\
-\sum_{k=1}^{N} \alpha_{2, k}\left[c_{i} \psi_{i k}-D \sum_{j=1}^{N} \int_{\Gamma_{j}} \phi^{*} \frac{\partial \psi_{k}}{\partial n} d \Gamma+\sum_{j=1}^{N} \int_{\Gamma_{j}}\left(\frac{\partial \phi^{*}}{\partial n}+\frac{\bar{v}_{n}}{D} \phi^{*}\right) \psi_{k} d \Gamma\right]
\end{gathered}
$$

Applying Eq.22 to all boundary nodes using a collocation technique, taking into account the previous functions, results in the following system of algebraic equations:

$$
H \phi-G q=(H \psi-G \eta) \alpha_{1}(t)+(H \psi-G \eta) \alpha_{2}(t)
$$

In the above system, the same matrices $H$ and $G$ are used on both sides. Matrices $\psi$ and $\eta$ are also geometrydependent square matrices (assuming, for simplicity, that the number of terms in Eq. 12 is equal to the number of boundary nodes), and $\phi, q$, and $\alpha$ are vectors of nodal values. The next step in the formulation is to find an expression for the unknown vectors $\alpha$. By applying expression 12 to all boundary nodes and inverting it and Eq. 18$]$, one arrives at:

$$
\alpha_{1}=F^{-1} \frac{\partial \phi}{\partial t}
$$

and

$$
\alpha_{2}=F^{-1}\left(P_{x}(x, y) \frac{\partial \phi}{\partial x}+P_{y}(x, y) \frac{\partial \phi}{\partial y}\right)
$$

which, substituted into 23) results in:

$$
H \phi-G q=(H \psi-G \eta) F^{-1} \frac{\partial \phi}{\partial t}+(H \psi-G \eta) F^{-1}\left(P_{x} \frac{\partial \phi}{\partial x}+P_{y} \frac{\partial \phi}{\partial y}\right)
$$

Calling:

$$
C=(H \psi-G \eta) F^{-1}
$$

gives

$$
H \phi-G q=C \frac{\partial \phi}{\partial t}+C\left(P_{x} \frac{\partial \phi}{\partial x}+P_{y} \frac{\partial \phi}{\partial y}\right)
$$

Next, we shall explain how to deal with the convective terms in Eq.28. 


\section{Handling convective terms}

In the present section, emphasis will be placed on the treatment of the convective terms. A mechanism must be established to relate the nodal values of $\phi$ to the nodal values of its derivatives. The approach will be discussed in detail in the next subsection.

\subsection{The Function-Expansion Approach}

This approach will start by expanding the values of $\phi$ at an internal point by using expression below:

$$
\phi=\sum_{k=1}^{M} \Im_{k} \beta_{k}
$$

where $\beta_{k}$ are constants. Now, by differentiating it with respect to $x$ and $y$ produces

$$
\frac{\partial \phi}{\partial x}=\sum_{k=1}^{M} \frac{\partial \Im_{k}}{\partial x} \beta_{k} \quad \text { and } \quad \frac{\partial \phi}{\partial y}=\sum_{k=1}^{M} \frac{\partial \Im_{k}}{\partial y} \beta_{k}
$$

Applying Eq. 29) at all $M$ nodes, a set of equations is produced that can be represented in matrix form by

$$
\phi=\Im \beta
$$

with corresponding matrix equations for expressions $(30)$ given as

$$
\frac{\partial \phi}{\partial x}=\frac{\partial \Im}{\partial x} \Im^{-1} \phi \quad \text { and } \quad \frac{\partial \phi}{\partial y}=\frac{\partial \Im}{\partial y} \Im^{-1} \phi
$$

Therefore, substituting Eq. 32 into Eq.(28), the new expression will be,

$$
(H-P) \phi-G q=C \frac{\partial \phi}{\partial t}
$$

where

$$
P=C\left(P_{x} \frac{\partial \Im}{\partial x}+P_{y} \frac{\partial \Im}{\partial y}\right) \Im^{-1}
$$

The coefficients of the diagonal perturbation matrix $P$ are all geometry-dependent only. The differential algebraic system (33) in time has a form similar to the one obtained using the finite element method (FEM) and hence, can be solved by any standard time integration algorithm by incorporating suitable modifications to account for its mixed nature. The system (33) can be integrated in time using time marching procedures [3]. It should be stressed that the coefficients of matrices $H, G$ and $C$ all depend on geometry only, thus they can be computed once and stored. In the forthcoming section, we shall explain how to solve this kind of algebraic system.

\section{Time marching schemes for the DRBEM formulation}

This section will show how to handle the linear algebraic system (33) adopting time marching schemes [1, 14, 15. A finite difference approximation for the time derivative term is given by:

$$
\frac{\partial \phi}{\partial t}=\frac{\phi^{i+1}-\phi^{i}}{\Delta t}
$$




$$
\begin{gathered}
\phi(t)=\left(1-\theta_{\phi}\right) \phi^{i}+\theta_{\phi} \phi^{i+1}, \\
q(t)=\left(1-\theta_{q}\right) q^{i}+\theta_{q} q^{i+1},
\end{gathered}
$$

Next, employing a general two-level time integration scheme for solution of Eq. (33), the following discrete form is obtained:

$$
\left[\frac{1}{\Delta t} C+\theta_{\phi}\{H-P\}\right] \phi^{m+1}-\theta_{\phi} G q^{m+1}=\left[\frac{1}{\Delta t} C-\left\{\left(1-\theta_{q}\right)(H-P)\right\}\right] \phi^{m}+\left(1-\theta_{q}\right) G q^{m}
$$

where $\phi^{m+1}$ and $q^{m+1}$ represent the potential and flux at the $(m+1)^{t h}$ time step, $\Delta t$ is the time step, $\phi^{m}$ and $q^{m}$ are the potential and flux at the $m^{\text {th }}$ time step, and $\theta$ is a relaxation parameter that determines if the method is explicit $\left(\theta_{\phi}, \theta_{q}=0\right)$ or implicit $\left(\theta_{\phi}, \theta_{q}=1\right)$. Several tests were done here to choose the best values for $\theta$ and we decided to select the backward difference scheme $\theta_{\phi}=1$ and $\theta_{q}=1$. In the time marching computation, the unknown quantities $\phi^{m}$ are updated at each time step by the new values obtained after solving Eq.(38). At the first time step, the temperature $\phi$ and heat flux $q$ at all boundary and internal points are specified with initial values. The computation ends when all time steps are fulfilled [16, 3] or a steady state is reached. The right side of Eq. 38 is known at all times. Upon introducing the boundary conditions at time $(m+1) \Delta t$, the left side of the equation can be rearranged and the resulting system solved by using standard direct procedures such as Gauss elimination and LU decomposition. More details of the element properties, interpolation functions, time integration and equation system formulation used in this paper are described in Brebbia et al. [5].

\section{Choice of radial basis functions}

In recent years, the theory of radial basis functions (RBFs) has undergone intensive research and enjoyed considerable success as a technique for interpolating multivariable data and functions. A radial basis function, $\Psi\left(x-x_{i}\right)=\psi\left(\left\|x-x_{j}\right\|\right)$ depends upon the separation distances of a sub-set of data centres, $X \subset \mathbb{R}^{n},\left\{x_{j} \in X, j=1,2, \ldots, N\right\}$. The distance, $\left\|x-x_{j}\right\|$, is usually taken to be the Euclidean metric, although other metrics are possible (for more details see Golberg and Chen [17]). The type of RBF used in the interpolation of the unknown variables normally plays an important role in determining the accuracy of the DRM [18. Partridge et al. [1] have shown that a variety of functions can in principle be used as global interpolation functions $f_{k}$. The approach used by Wrobel and DeFigueiredo [19] was based on practical experience rather than formal mathematical analyses and motivated by a previous successful experience with axisymmetric diffusion problems in which a similar approach was used [20]. In the present work, decision has been made to follow [19] by starting with a simple form of the particular solution $\psi$ and find the related expression for function $f$ by substitution directly into Eq. (14). The resulting expressions are

$$
\psi=r^{3},
$$




$$
\begin{gathered}
\eta=3 r\left[\left(x-x_{k}\right) n_{x}+\left(y-y_{k}\right) n_{y}\right] \\
f=9 D r-3 r\left[\left(x-x_{k}\right) v_{x}+\left(y-y_{k}\right) v_{y}\right]-k r^{3}
\end{gathered}
$$

in which $\left(x_{k}, y_{k}\right)$ and $(x, y)$ are the coordinates of the $k^{t h}$ boundary or internal point and a general point, $r$ but also on the diffusivity $D$, velocity components $v_{x}$ and $v_{y}$ as well as the reaction rate $k$, therefore, it will behave differently when diffusion or convection is the dominating process. The most popular RBFs are labelled as: $r^{2 m-2} \log r$ (generalised thin plate spline), $\left(r^{2}+c^{2}\right)^{m / 2}$ (generalised multiquadric) and $e^{-\beta r}$ (Gaussian) where $m$ is an integer number and $r=\left\|x-x_{j}\right\|$. Duchon 21] derived the thin plate splines (TPS) as an optimum solution to the interpolation problem in a certain Hilbert space via the construction of a reproducing kernel. It is interesting to observe that Duchon's thin plate splines function with $m=2$ corresponds to the fundamental solution commonly used in the BEM technique to solve biharmonic problems.

Another popular RBF for the DRM is the multiquadric (MQ). However, despite MQs excellent performance, it contains a free parameter, $c$, often referred to as the shape parameter and describes the relative 'flatness' of the RBFs about their centres. When $c$ is small the resulting interpolating surface is pulled tightly to the data points, forming a cone like basis functions. As $c$ increases, the peak of the cone gradually flattens. The Hardy multiquadric functions with values of $m=1$ and $c=0$ are often referred to as conical functions and, with $m=3$ and $c=0$, as Duchon cubic. Even though TPS have been considered optimal in interpolating multivariate functions, they do only converge linearly, Powell [22]. On the other hand, the multiquadric (MQ) functions converge exponentially as shown by Madych and Nelson [23]. The tuning of the free parameter $c$ can dramatically affect the quality of the solution obtained. Increasing the value of $c$ will lead to a flatter RBF. This will, in general, improve the rate of convergence at the expense of increased numerical ill-conditioning of the resulting linear system [23. Much effort has been made to search for the ideal shape parameter $c$ when utilising the MQ-RBF. This is due to the lack of information on choosing the best shape parameter available in the literature, forcing the user having to make an 'ad-hoc' decision. It is important to note that the value of the multiquadric shape parameter, $c$, has not been explicitly defined. It should also be noted that, following the procedures discussed in this section, the MQ-RBF is used to approximate the function $\psi$, not the function $f$. After a process of investigation, the authors found the optimal value of the non-dimensional shape parameter for the current problems to be $c=75$ and is used for all simulations.

The RBFs presented in table 1 have been examined in this paper. Thin-plate splines and the multiquadric are conditionally positive definite functions (for more details see [24]). 
Table 1: Radial Basis Functions

\begin{tabular}{c|c}
\hline \hline Name & Function \\
\hline \hline Multiquadric MQ & $\left(r^{2}+c^{2}\right)^{1 / 2}$ \\
\hline Thin Plate Spline TPS & $r^{2} \log r$ \\
\hline Cubic RBF & $r^{3}$ \\
\hline
\end{tabular}

\section{Numerical experiments and discussions}

The present section is concerned with the numerical application of the DRBEM for the solution of two-

The test results are obtained for Eq.(1) with the following initial and boundary conditions. The initial condition is chosen as the analytical value of Eq.410 for $t=0$ :

$$
\phi(x, y, t=0)=\sin (x)+\sin (y)
$$

Boundary conditions are chosen as:

$$
\begin{aligned}
& \phi(x=0, y, t)=(\sin (y)) e^{-2 t}, \phi(x=1, y, t)=(\sin (1)+\sin (y)) e^{-2 t} \\
& \phi(x, y=0, t)=(\sin (x)) e^{-2 t}, \phi(x, y=1, t)=(\sin (x)+\sin (1)) e^{-2 t}
\end{aligned}
$$

The analytic solution for the present case can be obtained from the following expression:

$$
\phi(x, y, t)=(\sin (x)+\sin (y)) e^{-2 t} .
$$

Figure 2 shows the geometrical mesh of the BEM model over a square channel. The boundary is discretised into 50 equally spaced constant elements per side. The analytical and the numerical solutions of this problem 


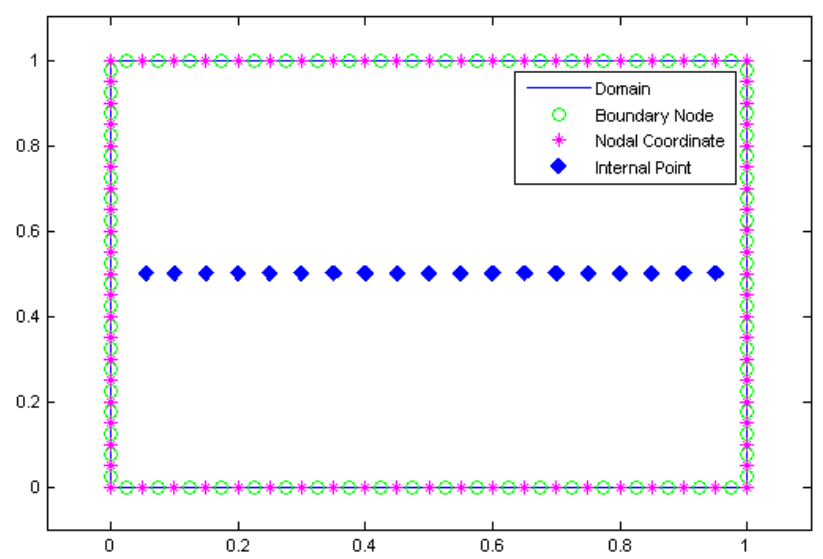

Figure 2: Geometrical mesh of convection- diffusion problem with side length $1 \mathrm{~m}$. when $\theta=1$ and time-step $\Delta t=0.05$. The result is obtained for the time evolution of the concentration profile along the centre line of the domain. Comparison between the above analytical solution and the numerical results show an excellent agreement.

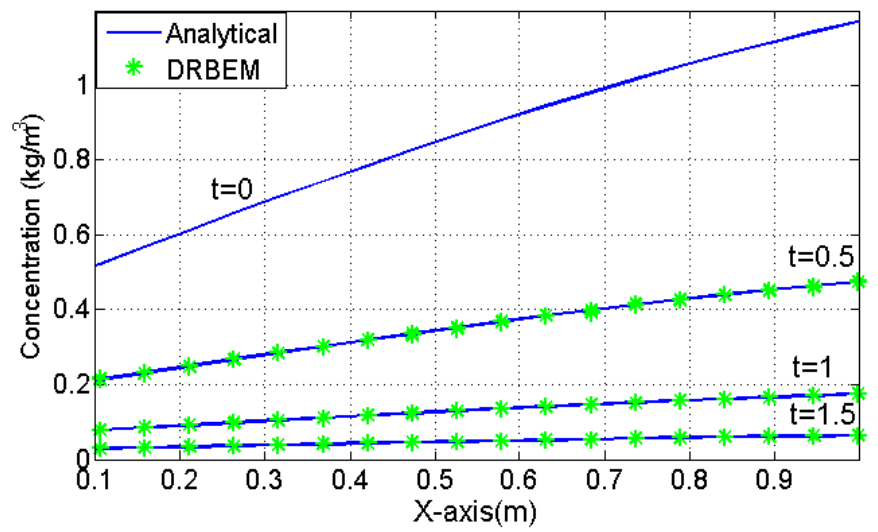

Figure 3: Concentration profile for every 10 time steps using MQ-RBF: comparison between the analytical (solid line) and numerical (star points) solutions.

Figures 4 and 5 consider the results using the thin-plate spline TPS-RBF and Cubic RBF also with time-step $\Delta t=0.05 \mathrm{~s}$. Similar results as for the MQ-RBF have been obtained in both cases. Figure 6 shows the time evolution of the concentration distribution in comparison with the analytical solution at the centre points of the computational domain i.e., $x=y=0.5$ using the backward-difference procedure and TPS-RBF. Table 2 shows a comparison between the three different RBFs with time-step value $\Delta t=0.05 \mathrm{~s}$ at time level $t=0.5$. It can be seen that the results obtained by the multiquadric, thin-plate spline and cubic RBFs are reasonably similar. In order to estimate the simulation error, the root mean square norm 


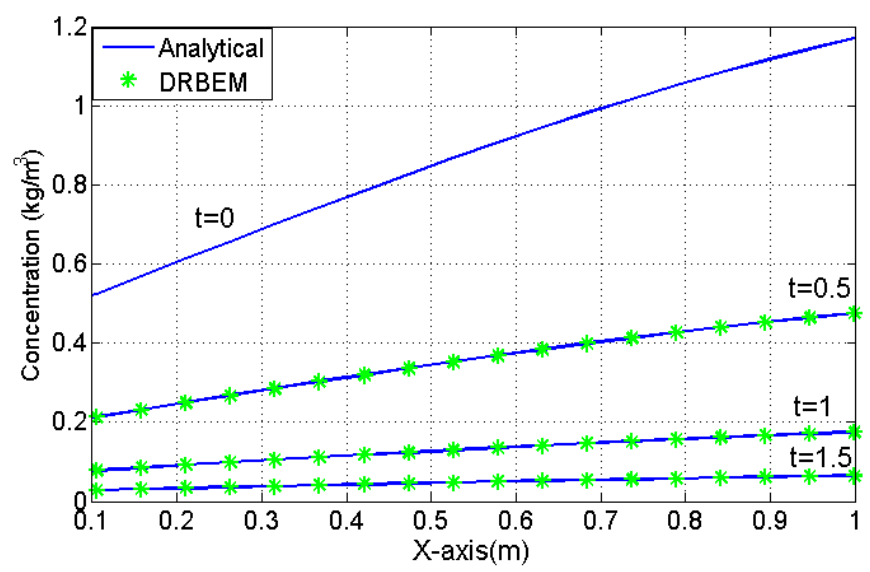

Figure 4: Concentration profile for every 10 time steps using TPS-RBF: comparison between the analytical (solid line) and numerical (star points) solutions.

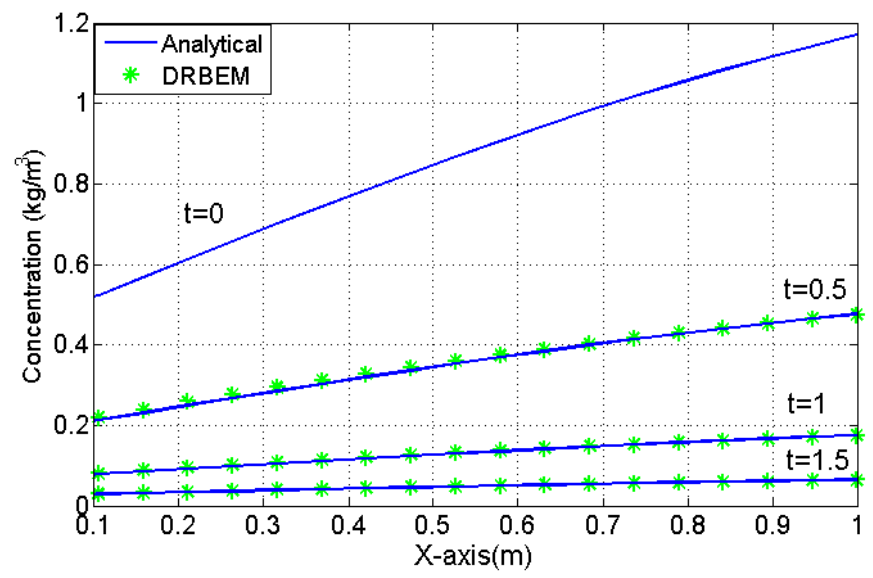

Figure 5: Concentration profile for every 10 time steps using Cubic-RBF: comparison between the analytical (solid line) and numerical (star points) solutions.

is utilised as shown in table 3. It is based on the difference between the simulation results $\phi_{\text {numer }}$ and the analytical solution $\phi_{\text {exact }}$ as

$$
R M S=\sqrt{\frac{1}{N} \sum_{i=1}^{N} \frac{\left(\phi_{i, \text { numer }}-\phi_{i, \text { exact }}\right)^{2}}{\phi_{i, \text { exact }}^{2}}},
$$

where $i$ denotes a nodal value, $\phi_{i, \text { exact }}$ is the analytical solution, $\phi_{i, n u m e r}$ is the numerical solution and $N$ is the total number of internal nodes, was computed for each analysis. In table 3 the error is seen to reduce as $\Delta t$ decreases, as expected. Table 4 shows a comparison between five different values of the shape parameter $c$ for MQ-RBF with time-step value $\Delta t=0.05 s$ at time level $t=0.5$. It is clear that the results obtained are reasonable and laying at same level of accuracy when the parameter $c=75$ or 100 . On the other hand, the results appear to lose their accuracy for smaller values of $c$.

From another point of view, taking a very high value of the shape parameter $c$ creates collocation matrices 


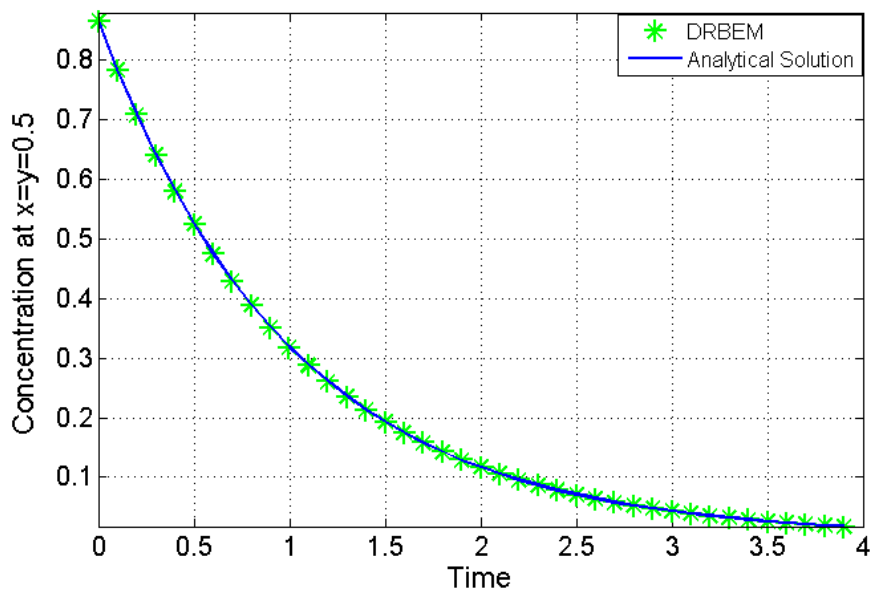

Figure 6: Concentration distribution with time using TPS-RBF: comparison of analytical (solid line) and numerical solution (star points) for at $x=y=0.5$.

Table 2: Results for convection-diffusion-reaction at $t=0.5$ for $\Delta t=0.05$

\begin{tabular}{lllll}
\hline $\mathrm{x}$ & Cubic & MQ & TPS & Analytical \\
\hline 0.055 & 0.1962 & 0.1939 & 0.2000 & 0.1965 \\
0.15 & 0.2304 & 0.2285 & 0.2395 & 0.2313 \\
0.25 & 0.2662 & 0.2680 & 0.2773 & 0.2673 \\
0.35 & 0.3013 & 0.3020 & 0.2951 & 0.3025 \\
0.50 & 0.3515 & 0.3490 & 0.3601 & 0.3527 \\
0.60 & 0.3829 & 0.3847 & 0.3893 & 0.3840 \\
0.75 & 0.4259 & 0.4258 & 0.4287 & 0.4271 \\
0.85 & 0.4517 & 0.4509 & 0.4535 & 0.4527 \\
0.95 & 0.4751 & 0.4759 & 0.4756 & 0.4756 \\
\hline
\end{tabular}

Table 3: RMS error of DRBEM at $t=2$ for decreasing $\Delta t$

\begin{tabular}{cccc}
\hline \multicolumn{4}{c}{$\theta=1, f=r^{2} \log (r)$, Problem 1} \\
\hline$\Delta t=0.1$ & $\Delta t=0.05$ & $\Delta t=0.025$ \\
\hline RMS error in $\phi$ & 0.0091 & 0.0067 & 0.0058 \\
\hline
\end{tabular}

which are poorly conditioned and require high-precision arithmetic to solve accurately. Using a relatively high non-dimensional shape parameter of 75 , the collocation matrices are sufficiently well conditioned to be solved using quad-precision arithmetic (see [25, 26, 27, for more details on the shape parameter $c$ ). 
Table 4: Results for convection-diffusion-reaction problem using MQ-RBF with different values of the shape parameter $c$

\begin{tabular}{lllllll}
\hline $\mathrm{x}$ & $\mathrm{c}=100$ & $\mathrm{c}=75$ & $\mathrm{c}=50$ & $\mathrm{c}=25$ & $\mathrm{c}=5$ & Analytical \\
\hline 0.055 & 0.1970 & 0.1939 & 0.1861 & 0.1852 & 0.3752 & 0.1965 \\
0.25 & 0.2678 & 0.2680 & 0.2468 & 0.2284 & 1.4938 & 0.2673 \\
0.50 & 0.3524 & 0.3490 & 0.3185 & 0.3025 & -3.5933 & 0.3527 \\
0.75 & 0.4268 & 0.4258 & 0.3984 & 0.3909 & -7.3263 & 0.4271 \\
0.95 & 0.4759 & 0.4759 & 0.4708 & 0.4662 & -1.6469 & 0.4756 \\
\hline
\end{tabular}

9.2. Two-dimensional transient convection-diffusion-reaction problem over a square channel with time-dependent

In order to further demonstrate the capability of the present numerical scheme for the solution of convection-diffusion-reaction with varying velocity values, the following problem is considered, where the velocities $v_{x}$ and $v_{y}$ are defined as:

$$
v_{x}(y)=\cos (\pi y), v_{y}(x)=-\cos (\pi x)
$$

The problem geometry, discretisation and interior nodes are schematically described in Fig. 2. The problem is modeled as square-shaped region, Dirichlet boundary conditions, and 19 internal nodes have been set at the middle line of the computational channel. Test results of this case study are obtained for the Eq.(1) with the following initial and boundary conditions. The initial condition is chosen as the analytical value of the Eq. 45 for $t=0$ :

$$
\phi(x, y, 0)=\sin (\pi x)+\sin (\pi y)
$$

Boundary conditions are chosen as:

$$
\phi(x=0, y, t)=\phi(x=1, y, t)=(\sin (\pi y)) e^{-D \pi^{2} t}, \phi(x, y=0, t)=\phi(x, y=1, t)=(\sin (\pi x)) e^{-D \pi^{2} t} .
$$

For the sake of convenience, we assume the diffusivity coefficient takes the value $D=1$, the reaction coefficient $k=0$. The problem is discretised into 200 equally spaced constant elements, 50 on each face. The analytical solution of the problem is given by

$$
\phi(x, y, t)=(\sin (\pi x)+\sin (\pi y)) e^{-D \pi^{2} t}
$$

Figure 7 shows the analytical and the numerical solutions of this problem using the cubic-RBF and implementing the fully implicit scheme when $\theta=1$ and time-step $\Delta t=0.005 \mathrm{~s}$. The results are obtained for the time evolution of the concentration profile along the centre line of the domain. The comparison between the above analytical solution and our numerical result shows excellent agreement. Figure 8 represents the numerical and exact solutions using the MQ-RBF, while Fig. 9 shows the numerical and exact solutions using the TPS-RBF, both with the same time-step $\Delta t=0.005 \mathrm{~s}$. Figure 10 illustrates the time evolution 


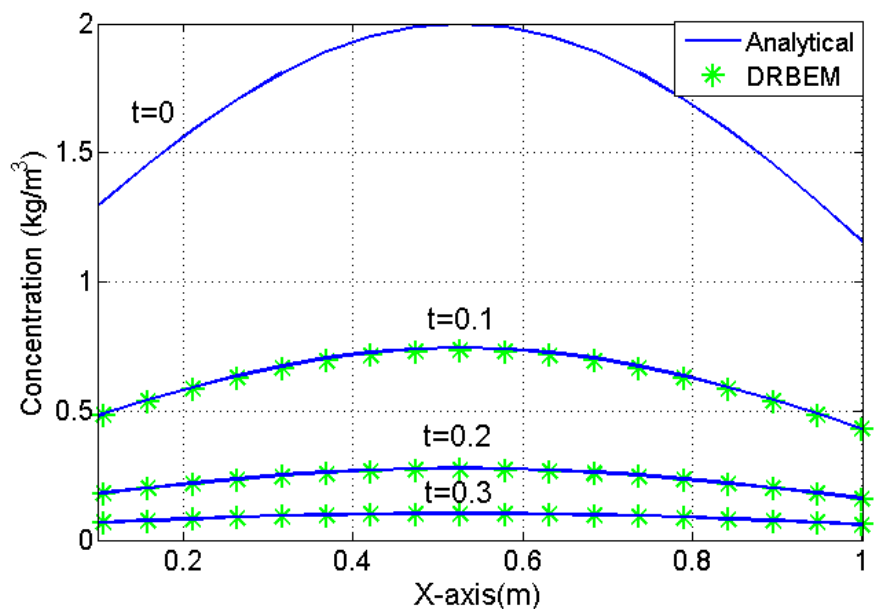

Figure 7: Concentration profile for every 20 time steps using Cubic-RBF: comparison between the internal analytical (solid line) and numerical (star points) solutions.

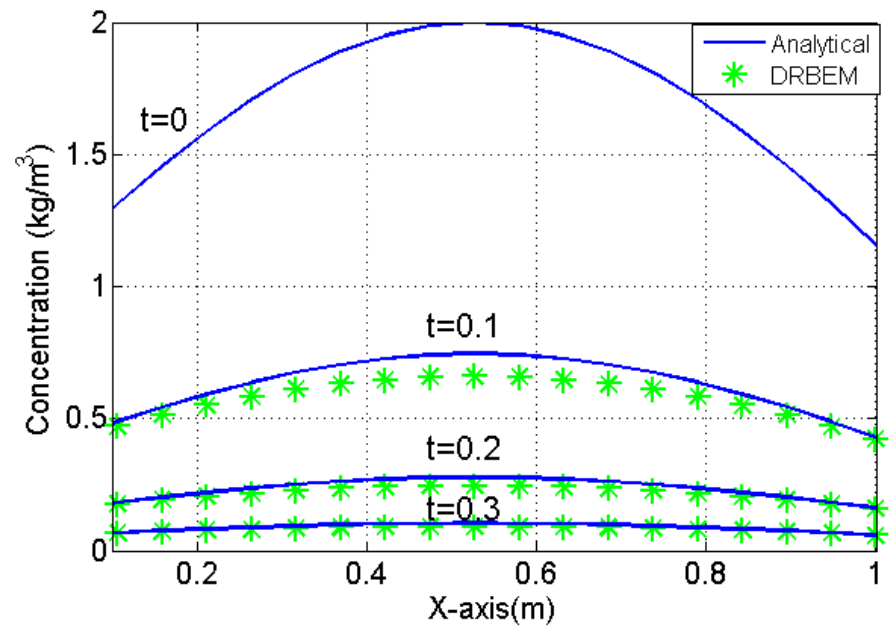

Figure 8: Concentration profile for every 20 time steps using MQ-RBF: comparison between the analytical (solid line) and numerical (star points) solutions.

of $\phi$ at $x=y=0.5$ along with the corresponding analytical solution using the thin-plate spline RBF. The numerical approximation for the propagation of $\phi$ with time is in close agreement with the analytical solution. The numerical error produced by the present numerical scheme decreases with propagating time. This guarantees the required numerical stability in the convection-diffusion problem. Thus, the salient properties of numerical solution for the consistency, stability, and accuracy are well treated in the present numerical experiments. As seen in table 5, the thin-plate spline and the cubic RBFs produce similar results, with good agreement with the analytical solution. In this case study, it is apparent that the accuracy is reduced with MQ-RBF. In table 6, the accuracy of the results for the best combination of parameters, $\Delta t=0.005$ and $f=r^{2} \log (r)$, is better than $0.01 \%$. 


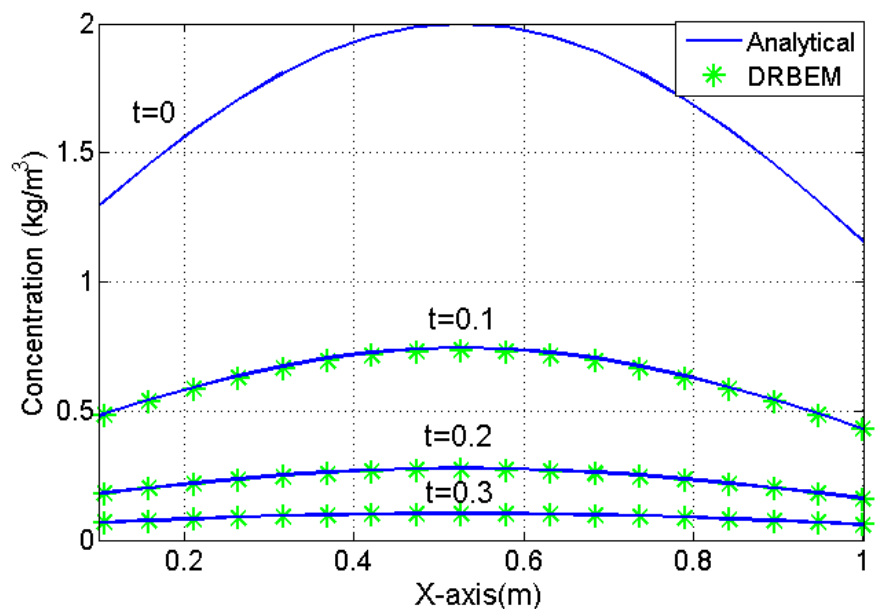

Figure 9: Concentration profile for every 20 time steps using TPS-RBF: comparison between the analytical (solid line) and numerical (star points) solutions.

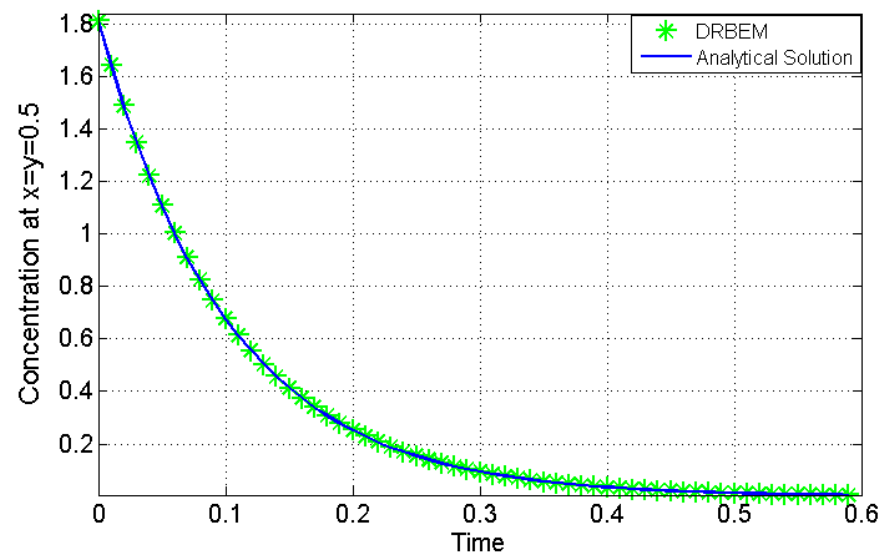

Figure 10: Concentration distribution of bounded domain: comparison for various time steps evolution of $\phi$ at $x=y=0.5$.

\subsection{Transient convection-diffusion-reaction problem over rectangular region with mixed (Neumann-Dirichlet)} boundary conditions

As a final example, we investigate a convection- diffusion problem with linear reaction term. The velocity field is considered to be along the longitudinal direction and all the coefficients in the governing equation are constant. The numerical and analytical solutions are compared for different time steps $\Delta t$ and reaction coefficient $k$. The geometry is considered to be $[0.7 m \times 1 m]$ as shown in Fig. 11. Potential values are imposed at the ends of the cross-section, i.e., at $x=0, \phi=300$ and at $x=1, \phi=10$. On the sides parallel to $x$, the zero lateral fluxes $q=0$, the problem thus having mixed Neumann-Dirichlet boundary conditions:

$$
\frac{\partial \phi}{\partial n}(x, 0, t)=\frac{\partial \phi}{\partial n}(x, 0.7, t)=0,0 \leq x \leq 1, t>0,
$$


Table 5: Results for convection-diffusion-reaction at $t=0.1$ for $\Delta t=0.005$

\begin{tabular}{lllll}
\hline $\mathrm{x}$ & Cubic & MQ & TPS & Analytical \\
\hline 0.055 & 0.434 & 0.428 & 0.444 & 0.436 \\
0.15 & 0.536 & 0.513 & 0.541 & 0.541 \\
0.25 & 0.627 & 0.583 & 0.633 & 0.636 \\
0.35 & 0.694 & 0.632 & 0.690 & 0.704 \\
0.50 & 0.733 & 0.660 & 0.722 & 0.745 \\
0.60 & 0.716 & 0.647 & 0.709 & 0.727 \\
0.75 & 0.628 & 0.583 & 0.637 & 0.636 \\
0.85 & 0.536 & 0.513 & 0.553 & 0.541 \\
0.95 & 0.429 & 0.423 & 0.439 & 0.431 \\
\hline
\end{tabular}

Table 6: RMS error of DRBEM at $t=2$ for decreasing $\Delta t$

\begin{tabular}{cccc}
\hline \multicolumn{4}{c}{$\theta=1, f=r^{2} \log (r)$, Problem 2} \\
\hline$\Delta t=0.01$ & $\Delta t=0.005$ & $\Delta t=0.0025$ \\
\hline RMS error in $\phi$ & 0.0153 & 0.0115 & 0.0097 \\
\hline
\end{tabular}

$$
\phi(0, y, t)=300, \phi(1, y, t)=10, \quad 0 \leq y \leq 0.7, t>0
$$

and the initial conditions are $\phi(x, y, 0)=0$ at all points at $t=0$. The values of the reaction parameter $k$ are assumed to be $k=1,5,10,20$, and $40 s^{-1}, v_{y}=0$ while $v_{x}$ is considered to vary according to the formula:

$$
v_{x}=k x+\log \left(\frac{10}{300}\right) x-\frac{k}{2}
$$

The steady-state solution is given in 10

$$
\phi(x, y)=300 \exp \left[\frac{k}{2} x^{2}+\log \left(\frac{10}{300}\right) x-\frac{k}{2} x\right]
$$

In the numerical simulation with a fully implicit scheme, a diffusion coefficient $D=1 \mathrm{~m}^{2} / \mathrm{s}$, a variable velocity $v_{x}$ as described in Eq.(48), and a time step $\Delta t=0.05 s$ were used. Comparison between the above analytical solution and our numerical results are given in figures below, showing excellent agreement.

Case (i): $k=1$ The first case is considered with the reaction value $k=1$, which is analysed considering the computational domain discretised into 80 constant elements and using 19 internal points with a time step $\Delta t=0.05 \mathrm{~s}$. For the DRBEM model, only the TPS-RBF has been applied in all cases. Figure 12 shows the exact and numerical solutions, with 10 constant elements along the vertical sides and 30 along each horizontal side.

Case (ii): $k=5$ In the second case, the contribution of the reactive term in Eq.(1) is increased to $k=5$. In Fig. 12, results are compared for the same time-stepping scheme considered in the previous case with 


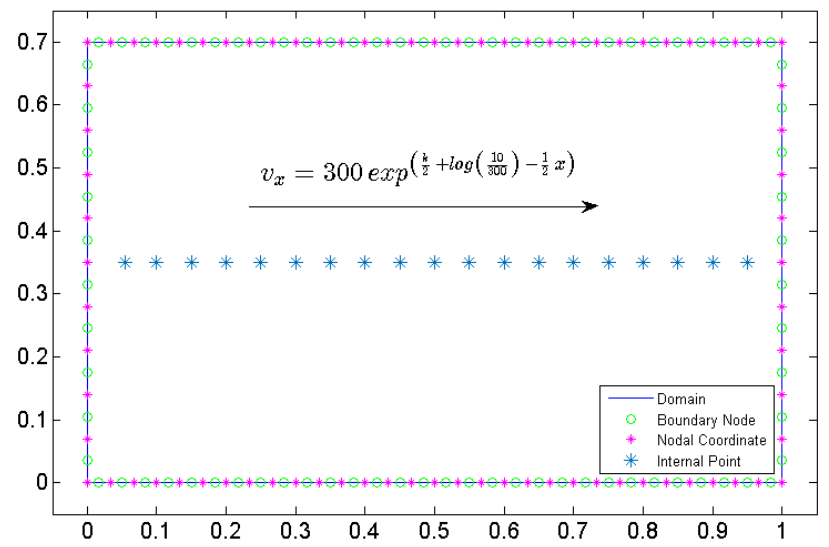

Figure 11: Schematic representation of the rectangular channel model with side length $1 \mathrm{~m}$.

$\Delta t=0.05$, at $t=2$ by which time the solution has converged to a steady-state. The results are still very reasonable for the discretisation employed, which is the same as for $k=1$.

Case (iii): $k=10$ For this case, the contribution of the reactive term in Eq.(1) is increased to $k=10$. Figure 12 displays the results time for the same time-stepping scheme considered in the previous case with $\Delta t=0.05$, at $t=2$ by which time the solutions have converged to a steady- state.

Case (iv): $k=20$ To see the effect of increasing the value of $k$, the reaction coefficient is now $k=20$. In this case, the maximum global Péclet number is equal to 10 (see, Fig. 13).

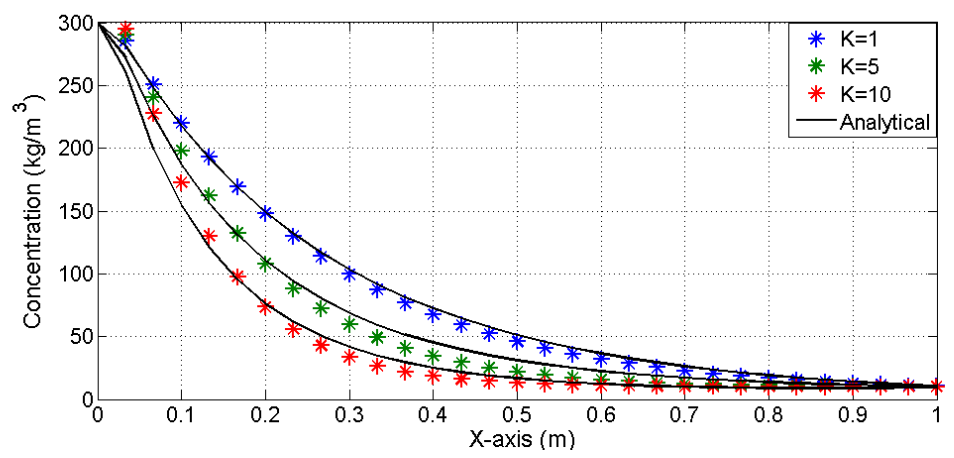

Figure 12: Concentration profile $\phi$ distribution for bounded domain with different values of reaction $k$ : Comparison between the analytical (solid line) and numerical (star points) solutions, for every 5 time steps, Problem 3.

Case (v): $k=40$ The final test considers the reaction coefficient $k=40$. A plot of the variation of the concentration $\phi$ along the $\mathrm{x}$-axis is presented in Fig. 13. In this case, the maximum global Péclet number is 20. It is obvious that the agreement with the corresponding analytical solution is still very good. 


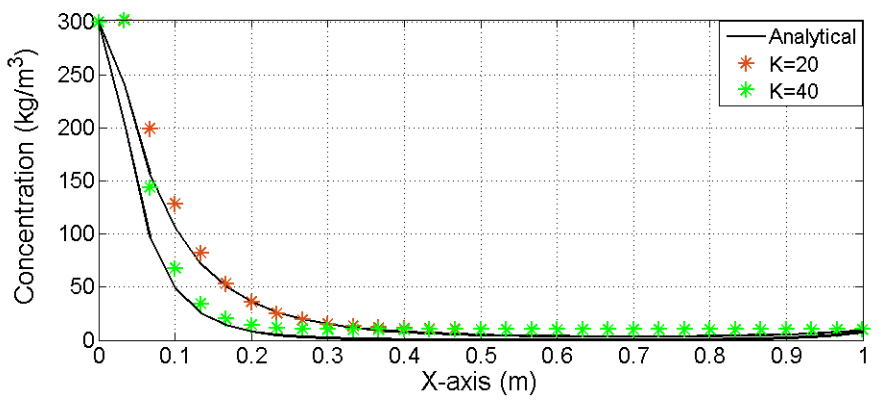

Figure 13: Comparison between the analytical (solid line) and numerical (star points) solutions, for different values of the reaction $k$.

\section{Conclusions and remarks}

In this paper, we present a novel formulation of the DRBEM for solving two-dimensional transient convection-diffusion-reaction problems with spatial variable velocity field. This new formulation for this type of problems has been implemented to handle the time derivative part and the variable velocity field. The fundamental solution of the corresponding steady-state equation with constant coefficients has been utilised. The DRBEM is used to transform the domain integrals appearing in the BEM formulations into equivalent boundary integrals, thus retaining the boundary-only character of the standard BEM. This formulation is expected to be stable at low Péclet numbers (i.e. diffusion-dominated problems). Numerical applications for $2 \mathrm{D}$ time-dependent problems are demonstrated to show the validity of the proposed technique, and its accuracy was evaluated by applying it to three tests with different velocity fields. Moreover, numerical results show that the DRBEM does not present oscillations or damping of the wave front as may appear in other numerical techniques.

In the empirical analysis section, the results presented in section 9 show the versatility of the method to solve time-dependent convection-diffusion-reaction problems involving variable velocity fields. The firstorder time derivative of the potential is approximated by employing a backward finite difference scheme. We can note a distinct advantage of the present approach, which demonstrates very good accuracy even for high reaction values which increase the Péclet number for the cases studied. It is obvious that, as the velocity increses, the concentration distribution becomes steeper and more difficult to reproduce with numerical models. However, all BEM solutions are still in good agreement for moderate Péclet number $($ Pé = 10 $)$ and (Pé $=20$ ), but oscillations appear for high Péclet number; thus, more refined discretisations are required. We have made an extensive investigation for the last case studied by considering many different values of the reaction coefficient $k$. For all these various values of $k$ the backward time-stepping scheme produces very good results in general. We have derived and implemented three RBFs and tested them with different types of problems, and we have found that the thin-plate spline is the most accurate among these RBFs for our problems. 


\section{Acknowledgments} (Al-Nahrain University) for the financial support and PhD scholarship.

\section{References}

[1] P. W. Partridge, C. A. Brebbia, L. C. Wrobel, The Dual Reciprocity Boundary Element Method, Comp. Mech. Pub., Southampton, 1992.

[10] P. W. Partridge, B. Sensale, The method of fundamental solutions with dual reciprocity for diffusion and diffusion-convection using subdomains, Engineering Analysis with Boundary Elements 24 (9) (2000) 633-641.

[11] G. D. Smith, Numerical Solution of Partial Differential Equations: Finite Difference Methods, Oxford

[12] L. C. Wrobel, D. B. DeFigueiredo, Numerical analysis of convection-diffusion problems using the boundary element method, International Journal of Numerical Methods for Heat \& Fluid Flow 1 (1) (1991) $3-18$. 
[13] J. Ravnik, L. Škerget, A gradient free integral equation for diffusion-convection equation with variable coefficient and velocity, Engineering Analysis with Boundary Elements 37 (4) (2013) 683-690.

[14] L. Cao, Q.-H. Qin, N. Zhao, Application of DRM-Trefftz and DRM-MFS to transient heat conduction analysis, Recent Patents on Space Technology 2 (2010) 41-50.

[15] E. Divo, A. J. Kassab, Transient non-linear heat conduction solution by a dual reciprocity boundary element method with an effective posteriori error estimator, in: ASME 2004 International Mechanical Engineering Congress and Exposition, American Society of Mechanical Engineers, 2004, pp. 77-86.

[16] K. Yang, X.-W. Gao, Radial integration BEM for transient heat conduction problems, Engineering Analysis with Boundary Elements 34 (6) (2010) 557-563.

[17] M. Golberg, C. Chen, The theory of radial basis functions applied to the BEM for inhomogeneous partial differential equations, Boundary Elements Communications 5 (2) (1994) 57-61.

[18] E. H. Ooi, V. Popov, Meshless solution of axisymmetric convection-diffusion equation: A comparison between two alternative RBIE implementations, Engineering Analysis with Boundary Elements 37 (4) (2013) 719-727.

[19] D. B. DeFigueiredo, Boundary Element Analysis of Convection-Diffusion Problems, PhD Thesis, Wessex Institute of Technology: Southampton, England (1990).

[20] J. C. F. Telles, A self-adaptive co-ordinate transformation for efficient numerical evaluation of general boundary element integrals, International Journal for Numerical Methods in Engineering 24 (5) (1987) 959-973.

[21] J. Duchon, Splines minimizing rotation-invariant semi-norms in Sobolev spaces, Constructive Theory of Functions of Several Variables, Springer, Berlin, Heidelberg, (1977) 85-100.

[22] M. Powell, The uniform convergence of thin plate spline interpolation in two dimensions, Numerische Mathematik 68 (1) (1994) 107-128.

[23] W. Madych, S. Nelson, Multivariate interpolation and conditionally positive definite functions. ii, Mathematics of Computation 54 (189) (1990) 211-230.

[24] P. Orsini, H. Power, M. Lees, The Hermite radial basis function control volume method for multizones problems; a non-overlapping domain decomposition algorithm, Computer Methods in Applied Mechanics and Engineering 200 (5) (2011) 477-493.

[25] A. H.-D. Cheng, Multiquadric and its shape parametera numerical investigation of error estimate, condition number, and round-off error by arbitrary precision computation, Engineering analysis with boundary elements 36 (2) (2012) 220-239. 
[26] D. Stevens, H. Power, C. Meng, D. Howard, K. Cliffe, An alternative local collocation strategy for highconvergence meshless PDE solutions, using radial basis functions, Journal of Computational Physics 254 (2013) 52-75.

[27] D. Stevens, H. Power, The radial basis function finite collocation approach for capturing sharp fronts in time dependent advection problems, Journal of Computational Physics 298 (2015) 423-445. 